\title{
Vertigem e síndrome antifosfolipídeo: como estão ligados?
}

\author{
Flavia P. Fleming, ${ }^{1 *}$ Roger A. Levy ${ }^{2}$
}

\begin{abstract}
Resumo
O objetivo dessa revisão é descrever a associação entre vertigem e a síndrome antifosfolipídeo (SAF), versando sobre seus achados clínicos e laboratoriais, com base na literatura científica. Já é visto na prática clínica e reconhecido pela comunidade científica que há uma associação entre a SAF e a vertigem. Sua principal apresentação clínica é na forma de síndrome de Meniere, com vertigem, hipoacusia sensorineural, plenitude aural e zumbido. Além dessa apresentação, sintomas audiovestibulares podem se apresentar da mais variada forma em pacientes com SAF. Devemos sempre lembrar que vertigem pode ser um sintoma central, principalmente em um paciente cuja doença de base gera tromboembolia de qualquer vaso, tanto do sistema arterial quanto venoso. Acidente vascular cerebral do sistema vertebrobasilar pode mimetizar um quadro vestibular periféfico. Nos pacientes com SAF e sintomas vestibulares, devemos ficar atentos ao avaliarmos a história, pois ela pode nos ajudar a diferenciar distúrbios centrais de periféricos. Exame físico otorrinolaringológico e neurológico completos são de suma importância. Portanto, as alterações da SAF levando a sintomas vestibulares podem ser tanto periféricas como centrais, essa última devendo sempre ser descartada, por ter maior gravidade, maior risco de complicação e sequela. Esses pacientes devem ter avaliação mutidisciplinar e vigilância constante.
\end{abstract}

Descritores: Vertigem; Síndrome antifosfolipídica; Doença de Meniere.

\section{Abstract \\ Vertigo and the antiphospholipid syndrome: how are they connected?}

The objective of this review is to describe the association between vertigo and the antiphospholipid syndrome (APS), talking about clinical and laboratory findings, based on scientific literature. It has been seen in clinical practice and recognized by scientific community that there is an association between the antiphospholipid syndrome and vertigo. Its main clinical presentation is Meniere's syndrome, with vertigo, sensorineural hearing loss, aural fullness and tinnitus. In addition to this presentation, audiovestibular symptoms may be presented in the most various ways in patients with APS. We should always have in mind that vertigo can be of central origin, especially in a patient whose disease can generate thrombosis in any blood vessel, either vein or artery. Vertebrobasilar stroke can mimic a peripheral vestibular syndrome. In APS patients with vestibular symptoms we should pay close attention to the case history, because it can
1. Serviço de Otorrinolaringologia. Hospital Universitário Pedro Ernesto. Universidade do Estado do Rio de Janeiro. Rio de Janeiro, RJ, Brasil.

2. Disciplina de Reumatologia. Departamento de Medicina Interna. Faculdade de Ciências Médicas. Universidade do Estado do Rio de Janeiro. Rio de Janeiro, RJ, Brasil.

*Endereço para correspondência:

Rua Visconde de Pirajá, 351/501

Rio de Janeiro, RJ, Brasil. CEP: 22410-906

E-mail: flaviapfleming@gmail.com

Revista HUPE, Rio de Janeiro, 2015;14(1):47-50

10.12957/rhupe.2015.14998

Recebido em 28/07/2014. Aprovado em 01/10/2014.

help us to differentiate peripheral and central disorders. A complete ENT and neurologic exam is very important. Thus, the alterations seen in APS that cause vestibular symptoms can be peripheral or central, this last one always has to be rolled out for its severity, risks of complication and sequela. These patients should have a multidisciplinary assessment and be closely monitored.

Keywords: Vertigo; Antiphospholipid syndrome; Meniere disease.

\section{Resumen}

Vértigo y síndrome del anticuerpo antifosfolipido: ¿cómo están relacionados?

El objetivo de esta revisión es describir la asociación entre el vértigo y el síndrome del anticuerpo antifosfolípido (SAF), tratándose sus hallazgos clínicos y de laboratorio, con base en la literatura científica.Ya es considerado en la práctica clínica y reconocido por la comunidad científica de que existe una asociación entre el SAF y el vértigo. Su principal presentación clínica es en forma de síndrome de Meniere, con vértigo, hipoacusia sensorioneural, plenitud aural y zumbido. Mas allá de esta presentación, síntomas audiovestibulares pueden presentarse de la forma más variada en pacientes con SAF. Debemos recordar siempre que el vértigo puede ser un síntoma central, especialmente en un paciente cuya enfermedad de base genera la tromboembolia de cualquier vaso, tanto en el sistema arterial como venoso. El accidente vascular cerebral del sistema vertebrobasilar puede simular un cuadro vestibular periférico. En los paciente con SAF con síntomas vestibulares, debemos 


\section{Artigo de revisão}

estar alerta al evaluar la historia, pues ésta puede ayudarnos a diferenciar disturbios centrales de periféricos. Los exámenes físico otorrinolaringológico y neurológico completos son de suma importancia.

Por lo tanto, las alteraciones del SAF que conducen a síntomas vestibulares pueden ser periféricas como centrales, esta última

\section{Introdução}

A síndrome antifosfolipídeo (SAF) é uma doença autoimune definida pela presença de eventos tromboembólicos recorrentes e/ou morbidade gestacional e de anticorpos antifosfolípides (aPL) incluindo anticardiolipina (aCL), anti-ß2-glicoproteína 1 (aß2GP1) e anticoagulante lúpico (LAC). Pode ser primária ou secundária, quando ocorre com outra doença autoimune, principalmente lúpus eritematoso sistêmico (LES). ${ }^{1}$ Qualquer vaso, seja arterial ou venoso, de qualquer calibre, inclusive microvascularização, pode ser afetado. ${ }^{2}$ Isso mostra que estamos diante de uma doença com uma gama muito ampla de apresentações clínicas. $\mathrm{O}$ fato de um paciente apresentar trombose tanto arterial quanto venosa deve chamar atenção, pois poucas trombofilias possuem essa característica. ${ }^{3}$

A principal apresentação venosa é a trombose venosa profunda de membro inferior e a principal apresentação arterial é o acidente vascular cerebral (AVC). Os critérios de classificação estão ilustrados no quadro 1. Para ser feito o diagnóstico, temos que ter um critério clínico (definido no consenso internacional) e um critério laboratorial, com duas comprovações sorológicas siempre debe descartarse, por ser de mayor gravedad, mayor riesgo de complicación y secuela. Esos pacientes deben tener evaluación multidisciplinar y vigilancia constante.

Palabras clave: Vértigo; Síndrome antifosfolípido; Enfermedad de Meniere.

em ocasiões diferentes, com um intervalo mínimo de 12 semanas, de acordo com protocolos pré-estabelecidos. ${ }^{3}$

Porém não é todo paciente que tem a presença de aPL que irá desenvolver fenômenos trombóticos. E, conforme exposto acima, não basta ter o aPL para ter o diagnóstico de SAF. O risco de trombose depende do anticorpo presente, sendo maior quando associado ao LAC e ainda maior se há os três anticorpos presentes. ${ }^{3}$ Portanto, a sorologia para SAF deve ser solicitada somente diante de suspeita clínica da doença ou de fator de risco como nos pacientes com LES. ${ }^{4}$

Os profissionais que trabalham com pacientes com SAF já tomaram conhecimento de que há uma relação entre essa doença e vertigem, porém a pesquisa do tema na literatura científica é muito escassa em todos os campos: epidemiologia, estudos clínicos e na área de ciência básica. A seguir será discutida essa associação.

\section{Funcionamento da orelha interna}

A orelha interna está localizada na porção petrosa do osso temporal, bilateralmente, e é dividida anatômica e funcionalmente em duas partes: o labirinto anterior e o labirinto posterior. A sua divisão anterior

\section{Quadro 1. Critérios de classificação da síndrome antifosfolipídeo.*}

\section{Critérios clínicos (um ou mais dos seguintes)}

- Trombose vascular: um ou mais episódios de trombose arterial, venosa ou de pequeno vaso em qualquer tecido ou órgão (confirmada por imagem ou histopatologia).

- Morbidade gestacional: uma ou mais mortes inexplicáveis de um feto morfologicamente normal com $\geq 10$ semanas de gestação; ou um ou mais nascimentos prematuros de um neonato morfologicamente normal antes de 34 semanas de gestação por causa de eclâmpsia, pré-eclâmpsia ou insuficiência placentária; ou três ou mais abortos espontâneos consecutivos e inexplicáveis antes de 10 semanas de gestação.

Critérios laboratoriais (um ou mais dos seguintes, presentes em duas ou mais ocasiões, com intervalo de, no mínimo, 12 semanas, usando procedimentos recomendados)

- LAC detectado de acordo com o guideline do ISTH.

- Anticorpo aCL isotipos IgG e/ou IgM, presentes em título médio ou alto (> 40 GPL ou MPL ou > percentil 99), utilizando ELISA padronizado.

- Anticorpo aß2GP1 isotipos IgG e/ou IgM em título > percentil 99, utilizando ELISA padronizado. 
contém a cóclea, responsável pela audição. Já a parte posterior compreende o vestíbulo (sáculo e utrículo) e os três canais semicirculares: anterior, posterior e horizontal. Essas cinco estruturas do labirinto posterior são responsáveis pela percepção do movimento e da posição da cabeça. Associadas à visão, à propriocepção e a diversos nervos, perifericamente, e junto com diversas estruturas e vias do sistema nervoso central (SNC), são responsáveis pelo equilíbrio. ${ }^{5}$

Essas áreas especializadas da orelha interna (labirinto anterior e posterior) possuem a mesma estrutura funcional, a célula ciliada. Ela transforma a energia mecânica do som e do movimento em energia elétrica. Através dessas células é passado o sinal elétrico para o oitavo par craniano, levando a mensagem ao SNC. Essas células estão imersas em um líquido rico em potássio, semelhante ao meio intracelular, chamado endolinfa. Essa composição eletrolítica permite a geração de um potencial elétrico para a despolarização das células ciliadas, quando estimuladas através da mobilização de seus cílios. ${ }^{5}$

Estudos anatômicos da orelha interna mostram que seu sistema capilar especializado é diferenciado e muito importante para seu funcionamento. Essa rica vascularização aí presente garante que a endolinfa tenha a característica eletrolítica já descrita, rica em potássio, polarização necessária para o funcionamento das células sensitivas. Esse rico fluxo sanguíneo da região também supre a energia gasta para manter esse ambiente. ${ }^{6}$ Além disso, a vascularização da orelha interna é terminal, suprida pela artéria labiríntica (artéria auditiva interna), na maioria das vezes, ramo da artéria cerebelar anteroinferior (AICA) - ramo da artéria basilar. A artéria labiríntica irriga a cóclea, a região vestibular inferior e superior separadamente, através de diferentes ramos. ${ }^{7}$ Portanto, em um paciente com SAF, pensando na fisiopatologia de sua doença de base, sintomas audiovestibulares podem vir de forma isolada ou em conjunto, como perda auditiva, vertigem, zumbido.

\section{Alterações na SAF}

Pesquisadores já descreveram que um grupo de sintomas são frequentes em pacientes com SAF, sintomas esses que compõem a síndrome de Meniere. ${ }^{8}$ São eles, além da vertigem (que pode estar associada a náuseas e vômitos), hipoacusia sensorineural (em geral, em baixas frequências), plenitude aural e zumbido. Nesse caso, a vertigem deve durar mais de 20 minutos, em geral horas, durando até dias e podendo vir acompanhada dos outros sintomas. ${ }^{9}$ Estudos de síndrome de Meniere mostram evidências de que o paciente afetado apresenta um volume elevado dentro do sistema endolinfático, mas a sua causa ainda é especulada. ${ }^{10}$ Outra característica é presença de sintomas flutuantes, em ataques. Apesar dos ataques de síndrome de Meniere parecerem completamente reversíveis. Ao longo do tempo e da repetição desses ataques, a orelha vai, gradualmente, perdendo a sua função, tanto da parte auditiva quanto da parte vestibular. ${ }^{11}$ Além de síndrome de Meniere, observamos as mais diversas alterações vestibulares, podendo estar associadas ou não a manifestações auditivas.

Além dessas alterações de origem periférica, não podemos esquecer, quando falamos de vertigem em SAF, de avaliar toda a via vestibular. Portanto, em um paciente com uma doença com um potencial vasto de alterações e de gravidade como esta, temos sempre que descartar que a vertigem seja de origem central, podendo inclusive ser mista.

A via vestibular central possui conexões no SNC, principalmente, no tronco encefálico e no cerebelo. ${ }^{5} \mathrm{~A}$ vascularização arterial dessa área é suprida pelo sistema vertebrobasilar. ${ }^{12}$ AVCs dessa localização podem ter a mais variada manifestação clínica, inclusive simulando uma síndrome vestibular periférica na abertura do quadro. ${ }^{12,13}$ Diante de um paciente com sintomas vestibulares, devemos atentar para a diferenciação de um quadro periférico e central. A história é muito importante, pois já nos direciona. Algumas informações das queixas do paciente são características. No exame físico, além do exame otorrinolaringológico, é crucial a avaliação neurológica completa, principalmente de pares cranianos e de função cerebelar. ${ }^{12}$ Podem ser necessários exames complementares para guiar um diagnóstico mais preciso.

\section{Conclusão}

A SAF foi descrita pela primeira vez em $1983,{ }^{8}$ sendo uma doença nova e ainda preservando muitos mistérios, com vastos campos a serem estudados pela medicina.

Em um paciente com SAF, podemos prever quantos distúrbios de orelha interna podem haver se algum dos mecanismos de lesão de sua doença vier a alterar o funcionamento tão harmônico da plena função audiovestibular. Perifericamente, especialmente distúrbios microtrombóticos, podem causar não só vertigem isolada, como perda auditiva, o espectro completo de sintomas da síndrome de Meniere, entre outras variadas 


\section{Artigo de revisão}

combinações de sintomas de orelha interna.

Nesses pacientes, devemos ficar alertas quando estamos diante de sintomas audiovestibulares, pois uma síndrome vestibular periférica pode mascarar patologias de origem central. Todo paciente com sintomas vestibulares deve ser cuidadosamente avaliado. Atenção especial para a história, pois ela pode ajudar a nos guiar.

Pacientes com SAF devem ter acompanhamento periódico reumatológico. Caso sintomas específicos audiovestibulares ocorram, a avaliação conjunta com o otorrinolaringologista é essencial. Sempre na suspeita de origem central, é importante a avaliação de um neurologista.

\section{Referências}

1. Comarmond C, Cacoub P. Antiphospholipid syndrome: from pathogenesis to novel immunomodulatory therapies. Autoimmun Rev. 2013;12:752-757. http://dx.doi.org/10.1016/j. autrev.2012.12.006

2. Hughes GRV, Cuadrado MJ. Hughes (antiphospholipid) syndrome: clinical features. Rheum Dis Clin North Am. 2001;27(3):507-524.

3. Lim W. Antiphospholipid syndrome. Hematology Am Soc Hematol Educ Program. 2013; 2013:675-680.

4. Arachchillage DJ, Cohen H. Antiphospholipid syndrome. Medi- cine (Abingdon). 2014; 42(3):156-161.

5. Flint PW, Haughey $\mathrm{BH}$, Lund VJ, et al. Cummings Otolaryngology - Head and Neck Surgery. 5th edition. Philadelphia, PA: Mosby/Elsevier; 2010. 2963p.

6. Patuzzi R. Ion flow in stria vascularis and the production and regulation of cochlear endolymph and the endolymphatic potential. Hear Res. 2011;277:4-19. http://dx.doi.org/10.1016/j. heares.2011.01.010

7. Nakashima T, Naganawa S, Sone M et al. Disorders of cochlear blood flow. Brain Res Rev. 2003;43:17-28. http://dx.doi. org/10.1016/S0165-0173(03)00189-9

8. Hughes GRV. Hughes syndrome (the antiphospholipid syndrome): a disease of our time. Inflammopharmacol. 2011;19:69-73. http://dx.doi.org/10.1007/s10787-010-0071-3

9. Monsell EM, Balkany TA, Gates GA, et al. Committee on hearing and equilibrium guidelines for the diagnosis and evaluation of therapy in Meniere's disease. Otolaryngol Head Neck Surg. 1995;113:181-185.

10. Agrawal Y, Minor LB. Physiologic effects on the vestibular system in Meniere's disease. Otolaryngol Clin N Am. 2010;43:985993. http://dx.doi.org/10.1016/j.otc.2010.05.002

11. Foster CA, Breeze RE. The Meniere attack: an ischemia/ reperfusion disorder of inner ear sensory tissues. Med Hypotheses. 2013;81:1108-1115. http://dx.doi.org/10.1016/j. mehy.2013.10.015

12. Edlow JA, Newman-Toker DE, Savitz SI. Diagnosis and initial management of cerebellar infarction. Lancet Neurol. 2008;7:951964.

13. Lee $\mathrm{H}$. Audiovestibular loss in anterior inferior cerebellar artery territory infarction: a window to early detection? J Neurol Sci. 2012;313:153-159. http://dx.doi.org/10.1016/j.jns.2011.08.039 